\title{
Botulinum Therapy in the Early Regeneration Period after Blepharoplasty
}

\author{
Max-Adam Scherer* \\ Plastic surgeon, Dermatologist, Cosmetologist Clinic of High Aesthetic Medicine Moscow, Russia.
}

Received: May 17, 2017; Accepted:June 09, 2017; Published: June 16, 2017

*Corresponding author: Max-Adam Scherer, Plastic surgeon, dermatologist, cosmetologist Clinic of High Aesthetic Medicine Moscow, Russia, E-mail: pro.yulia2012@yandex.ru

\begin{abstract}
The author presents his method of using BTA injections in the periorbital area for promoting the wound healing process, ensuring formation of a normotrophic scar and enhancing the aesthetic outcome of blepharoplasty surgeries.
\end{abstract}

Keywords: BTA injections; Periorbital Area Rejuvenation; Normortophic Scar; Regeneration after Blepharoplasty.

\section{Introduction}

In the course of many years various blepharoplasty techniques proved to be effective for surgical rejuvenation of the periorbital area.

Success of any blepharoplasty depends - among other factors - upon the normal course of the wound healing process. Surgeons strive to create favorable conditions for wound healing and to minimize the expected side effects (postsurgical edema, hematomas etc.). And the main objective, of course, is to provide formation of a normotrophic scar. BTA-induced chemoimmobilization of facial muscles in this area even after removal of sutures and retaining bandages eliminates dynamic forces disturbing the healing wound. Moreover, results of some recent research suggest that the presence of BTA in the tissues of healing cutaneous wounds can inhibit processes leading to scar hypertrophy $[1,2,3]$.

It is also necessary to bear in mind, that the patients usually expect a full rejuvenation after blepharoplasty, including smoothing of dynamic wrinkles as well. BTA injections after the operation represent a good solution.

Being guided by the patients' needs and considering the options of botulinum therapy, I began to use BTA injections in the early postoperative period and worked out a management algorithm of my own for patients after various types of blepharoplasty.

\section{General considerations}

The following timing options for applying botulinum therapy as a procedure for enhancing the outcome of a face or neck plastic surgery are usually considered:

tients were clinically diagnosed with solar lentigo or solar lentigines (Fig.1, 3, 5, 7, 9, 12, 15).

10 - 20 days before surgery [4];

- intraoperatively (injections into the orbicularis oculi muscle under direct visualization or immediately after wound closure) $[5,6,7,8,9,10,11]$;

a in the early postoperative period (24 - 96 hours after the operation) $[12,13,14,15,16]$;

several months postoperatively.

If botulinum therapy is accomplished $10-20$ days before blepharoplasty, a particular lifting effect is present in the facial soft tissues at the time of surgery. This may interfere with exact evaluation of the amount of skin to be excised. Furthermore, BTA injections in the area under the eyes may also lead to infraorbital hernia accentuation or prolapse.

Botulinum therapy may be accomplished intraoperatively, but I regard this as questionable: many surgeons use soft tissue hydro dissection or local anesthesia with epinephrine before blepharoplasty. This may influence the process of BTA fixation in the muscles, thus weakening the injections effect.

Several months after blepharoplasty active wound healing is already accomplished, and botulinum toxin injections can not affect this process any more. At this point BTA injections can be considered only as a traditional means of expressive facial wrinkles correction.

In my opinion the optimal time for applying botulinum therapy after blepharoplasty in order to enhance the wound healing process and aesthetic outcomes of a blepharoplasty is the early postoperative period (the 3rd, 5th or 7 th day after surgery) 
while other timing options are associated with substantial drawbacks. The reasons from my viewpoint as a practitioner are the following:

1) Chemoimmobilization of the orbicularis oculi muscle allows to create the most favorable conditions for excellent apposition of wound edges, to eliminate dynamic tension and soft tissue motility in the area of the healing wound, thus ensuring formation of a normotrophic scar and preventing hypertrophic scarring;

2) $[1,2]$ The observed in vitro inhibiting effect of BTA on fibroblast differentiation and the BTA-induced decrease in CTGF proteins in hypertrophic scar-derived cell cultures suggest an additional prophylactic action of BTA regarding cutaneous scar hypertrophy. So every opportunity should be used to ensure formation of an inconspicuous scar;

3) Elimination or substantial reduction of dynamic wrinkles (meeting the patients' expectations);

4) Use of botulinum therapy in the early postoperative period after blepharoplasty allows to ensure high

effectiveness of BTA injections as a means of surgery outcome enhancement and avoid drawbacks associated with other timing options.

The chemoimmobilizing effect of BTA injections on facial muscles and its safe use for aesthetic correction of facial rhytids and mild soft tissue ptosis is very well established scientifically and clinically $[17,18,19,20]$. A group of researchers showed that botulinum toxin type A directly inhibited fibroblast-tomyofibroblast differentiation in a cell culture derived from hypertrophic scars, but they observed no such effect in a culture derived from normotrophic scars [1]. Other researchers noted an in vitro decrease in connective tissue growth factor (CTGF) protein in fibroblasts derived from hypertrophic scars due to BTA action [2] and also showed an inhibiting action of BTA on collagen deposition in a rabbit model of hypertrophic scarring [3]. The results of such studies provide theoretical support for use of BTA injections to prevent/control hypertrophic scarring.

Nowadays various assumptions arising from practitioners' observations of BTA injection effects on the cutaneous wound healing process prompt a lot of potentially interesting topics for future detailed studies. For example, in my practice I observe a weaker myoimmobilizing effect of BTA injections on the orbicularis oculi muscle made in the early regenerative period after blepharoplasty compared to usual aesthetic BTA correction of intact facial soft tissues. Further research is necessary to understand the exact mechanism of BTA effects on actively regenerating soft tissues of cutaneous wounds.

\section{Marking}

Marking of the injection site is an important step that ensures achievement of the planned result. I have developed a marking scheme (Figure 1) that ensures reproducibility of the method and predictability of the outcome.

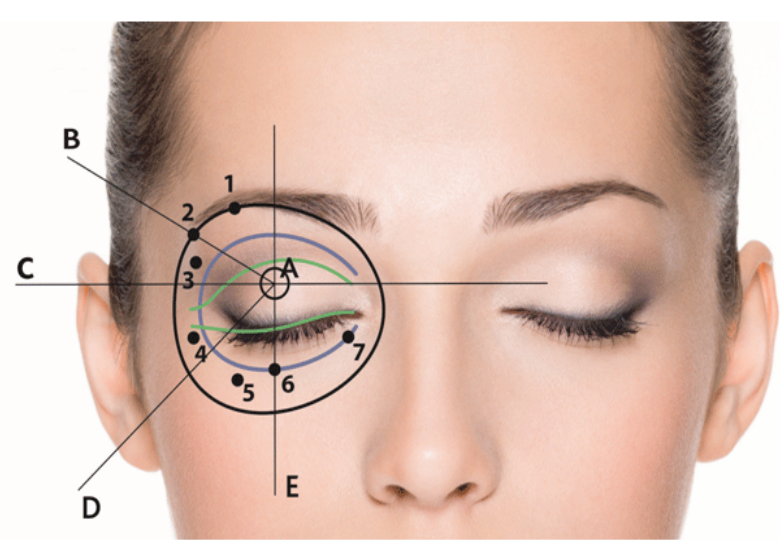

Figure 1: Marking of the periorbital zone before BTA injections.

- point $\mathrm{A}$ - the pupil center;

- black line - the outer border of the orbicular muscle of the eye (m. orbicularis oculi);

- blue line - the orbital border of the orbicular muscle of the eye (the bony landmark);

- green line - the blepharoplasty skin incision path;

- $\mathrm{AB}$ - the line from the pupil center to the brow tail end;

- AC - the horizontal transpupil line;

- $\mathrm{AD}$ - the line from the pupil center to the earlobe;

- AE - the vertical transpupil line;

- 1 - the brow arch maximal curvature point

- 2 - the point on the brow tail end;

- $3,4,5$ - the points in the centers of segments formed by lines $\mathrm{AB}, \mathrm{AC}, \mathrm{AD}, \mathrm{AE}$;

- 6 - the intersection line $\mathrm{AE}$ with the inferior part of the line indicating the orbital border of the orbicular muscle of the eye;

- 7 - the additional point in the area of the inner corner of the eye on the medial inferior part of the line indicating the orbital border of the orbicular muscle of the eye.

Let's denote the center of the pupil of the eye as point A. Four straight lines are to be drawn virtually through this point: the horizontal transpupil line $\mathrm{AC}$, the line $\mathrm{AB}$ - through the pupil center and the endpoint $B$ of the browtail; the line $A D$ - from the pupil center towards the earlobe; and the vertical transpupil line $\mathrm{AE}$.

Then we locate the injection points, denoting them with numbers:

1 - the brow arch maximal curvature point (in women I introduce BTA preparations into a point under the brow , and in men - into a point right in the brow);

2 - the point on the brow tail end;

$3,4,5$ - the points in the centers of segments formed with lines $\mathrm{AB}, \mathrm{AC}, \mathrm{AD}, \mathrm{AE}$; 
6 - the intersection of the transpupil line $\mathrm{AE}$ with the inferior part of the line indicating the inner border of the orbicular muscle of the eye (in Figure 1 the blue line shows the inner border of the orbicular muscle of the eye and the black line shows the outer border of the orbicular muscle of the eye);

7 - the point in the area of the inner corner of the eye on the inferior medial part of the line indicating the inner border of the orbicular muscle of the eye (for an extra injection if necessary).

\section{Technique and dosages}

Any BTA preparation may be used for this method, the dilution and dosage data is given for Botox (Allergan, USA) as an example. BTA introduction modes: intramuscular injections of a classically diluted solution (100 U BTA and $2 \mathrm{ml}$ saline) and intradermal injections in nappage technique of a less concentrated solution (100 U BTA and $4 \mathrm{ml}$ saline).

BTA injections into points 1, 6 and 7 are made intradermally or subdermally in papula technique, $0,5 \mathrm{U}$ for each point. It is necessary to achieve mild relaxation of the orbicular muscle of the eye and to immobilize the lower eyelid in the incision area, so small doses of botulinum toxin are injected. (The green line in Figure 1 shows the incision path)

Botulinum toxin injections into points 2, 3, 4, 5 are made intramuscularly, $1 \mathrm{U}$ for each point. Point 7 is used for an additional injection $(1 \mathrm{U})$, if extra correction is necessary for immobilization of the orbicular muscle of the eye.

Botulinum toxin injections along the incision path (at the wound edges) in nappage technique are made to enhance the regeneration process. $1-0,5 \mathrm{U}$ (depending on the incision length) of a classical solution is diluted $1: 1$ with $0,9 \% \mathrm{NaCl}$ directly in an additional syringe.

For male faces I use the same marking scheme and similar doses as for female patients, but there are some specific details. I usually do not inject BTA into points 2 and 3 in the segment between lines $\mathrm{AB}$ and $\mathrm{AC}$ to avoid brow tail rise. An elevated brow tail brings an air of femininity to male features and is therefore undesirable. I inject BTA into points 2 and 3 in men only if a correction of mild brow ptosis for additional enhancement of a lower blepharoplasty surgery outcome is necessary or in some cases when creation of favorable conditions for wound healing after lower or all-round blepharoplasty has overriding priority. In regard to BTA dosages my vast practice of BTA use for noninvasive aesthetic facial correction proves absolute sufficiency of standard BTA doses for men [21].

The method shows good results: in the course of 2 years I used botulinum therapy in the early period after blepharoplasty in 55 patients, the planned outcome was achieved in all cases, no adverse effects were observed, patient satisfaction was high.

\section{Case report}

A female patient B, 39 years old, presented in my clinic with complaints about skin excess in the upper eyelids (Figures 2 and 3: Before blepharoplasty). An upper blepharoplasty was performed. On the day of suture removal BTA injections in the periorbital area were made ( Figures 4 and 5: 7 days after upper blepharoplasty, markings for BTA injections). Figure 6 shows the patient 2 months after surgery.
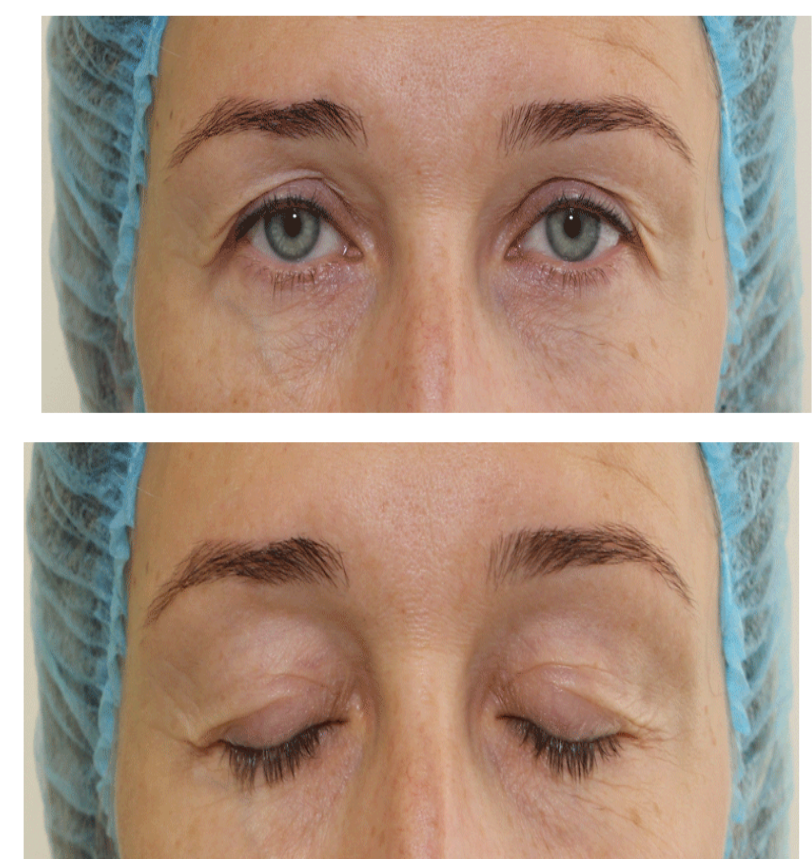

Figure 2 \& 3: Before Blepharoplasty
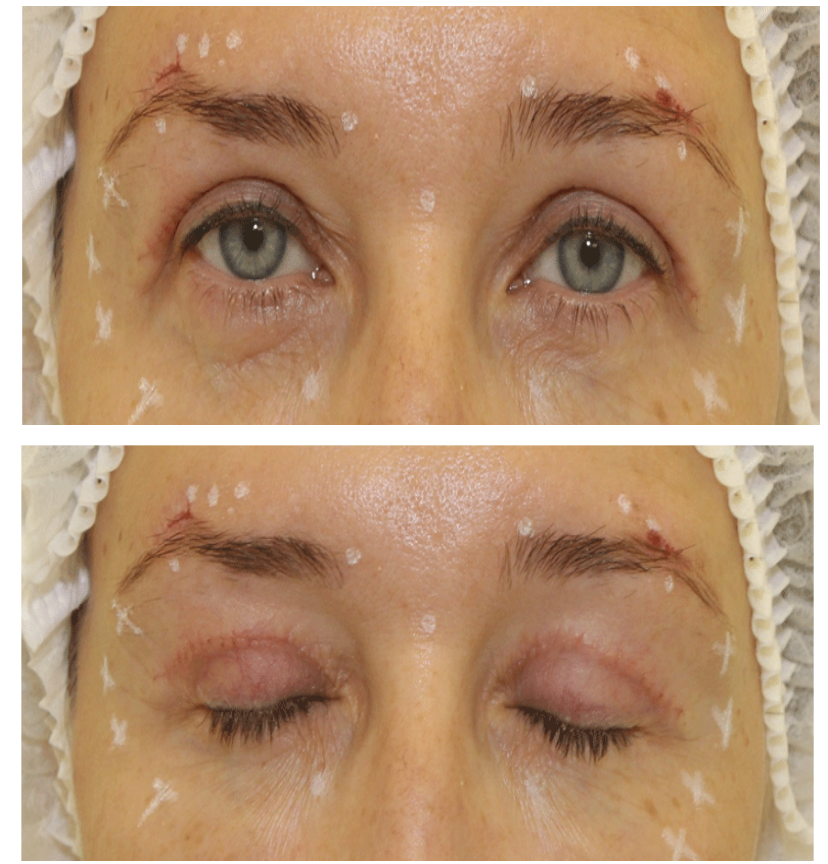

Figure 4 \& 5: 7 Days after upper Blepharoplasty, markings for BTA injections 


\section{Conclusion}

So, the advantages of botulinum therapy in the early rehabilitation period after blepharoplasty are obvious. It ensures favorable conditions for wound healing, formation of a normotrophic scar, acts as a preventive agent against hypertrophic scarring, and allows eliminating or significantly lessening wrinkles in the periorbital zone. So the blepharoplasty patients' expectations are met in full.

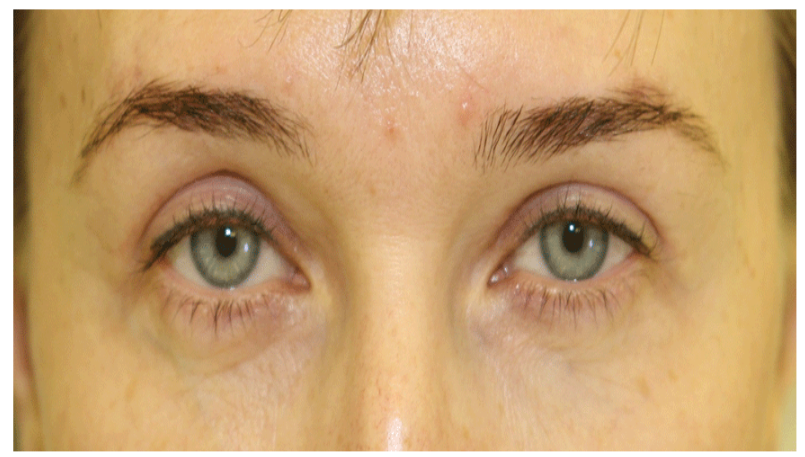

Figure 6: Shows the patient 2 months after surgery

\section{References}

1. Jeong HS, Lee BH, Sung HM, Park SY, Ahn DK, Jung MS, et al. Effect of Botulinum Toxin Type A on Differentiation of Fibroblasts Derived from Scar Tissue. Plast Reconstr Surg. 2015;136(2):171e-178e. doi: 10.1097/PRS.0000000000001438

2. Xiao Z, Zhang M, Liu Y, Ren L. Botulinum toxin type a inhibits connective tissue growth factor expression in fibroblasts derived from hypertrophic scar. Aesthetic Plast Surg. 2011;35(5):802-807. doi: 10.1007/ s00266-011-9690-3

3. Xiao Z, Qu G. Effects of botulinum toxin type a on collagen deposition in hypertrophic scars. Molecules. 2012;17(2):2169-2177. doi: 10.3390/molecules17022169

4. Serra-Renom J-M, Serra-Mestre J-M. Frontal, temple and perioirbital fat grafting. In: Atlas of Minimally Invasive Facelift (Facial rejuvenation with volumetric lipofilling), Serra-Renom J-M, Serra-Mestre J-M, Springer 2016; Chapter 8, p. 49.

5. Guerrissi JO. Intraoperative Injection of Botulinum Toxin A into Orbicularis Oculi Muscle for the Treatment of Crow's Feet. Plast Reconstr Surg. 2000;104(6):2219-2225.

6. Choi JC, Lucarelli MJ, Shore JW. Use of botulinum A toxin in patients at risk of wound complications following eyelid reconstruction. Ophthal Plast Reconstr Surg. 1997;13(4):259-264. DOI: 10.1097/00002341199712000-00006

7. Sherris DA, Gassner HG. Botulinum toxin to minimize facial scarring. Facial Plast Surg. 2002;18(1):35-39.
8. Gassner HG, Sherris DA. Chemoimmobilization: improving predictability in the treatment of facial scars. Plast Reconstr Surg. 2003;112(5):1464-1466.

9. Wilson AM. Use of botulinum toxin type A to prevent widening of facial scars. Plast Reconstr Surg. 2006;117(6):1758-1766.

10. Flynn TC. Use of intraoperative botulinum toxin in facial reconstruction. Dermatol Surg. 2009;35(2):182-188. doi: 10.1111/j.15244725.2008.34407.x

11. Malik A, Gupta PC, Ram J. Wound Modulation with Botulinum Toxin. J Ophthalmic Vis Res. 2016;11(2):240-241. doi: 10.4103/2008322X.183919

12. Gassner HG, Brissett AE, Otley CC, Boahene DK, Boggust AJ, Weaver AL, et al. Botulinum toxin to improve facial wound healing: A prospective, blinded, placebo-controlled study. Mayo Clin Proc. 2006;81(8):10231028.

13. Gassner HG, Sherris DA, Friedman O. Botulinum toxin-induced immobilization of lower facial wounds. Arch Facial Plast Surg. 2009;11(2):140-142. doi: 10.1001/archfacial.2009.3

14.Ziade M, Domergue S, Batifol D, Jreige R, Sebbane M, Goudot P, et al. Use of botulinum toxin type $A$ to improve treatment of facial wounds: a prospective randomized study. J Plast Reconstr Aesthet Surg. 2013;66(2):209-214.

15. Jablonka EM, Sherris DA, Gassner HG. Botulinum toxin to minimize facial scarring. Facial Plast Surg. 2012;28(5):525-535.

16. Kim YS, Lee HJ, Cho SH, Lee JD, Kim HS. Early postoperative treatment of thyroidectomy scars using botulinum toxin: a split-scar, double-blind randomized controlled trial. Wound Repair Regen. 2014;22(5):605-612. doi: 10.1111/wrr.12204

17. Kane M, Sattler G. Illustrated guide to aesthetic botulinumtoxin injections: Basics, Localization, Uses (Aesthetic Methods for Skin Rejuvenation) Quintessence Publishing Co. Ltd, New Maiden, UK. 2014.

18. Jost WH. Pictorial atlas of botulinum toxin injection: Dosage, Localization, Application" /2nd Revised edition/ Quintessence Publishing Co. Ltd, New Maiden, UK. 2013.

19. Cavallini M, Cirillo P, Fundarò SP, Quartucci S, Sciuto C, Sito G, et al. Safety of botulinum toxin A in aesthetic treatments: a systematic review of clinical studies. Dermatol Surg. 2014;40(5):525-536. doi: 10.1111/dsu. 12463

20.Sundaram H, Signorini M, Liew S, Trindade de Almeida AR, Wu Y, Vieira Braz A, et al. Global Aesthetics Consensus Group. Global Aesthetics Consensus: Botulinum Toxin Type A - Evidence-Based Review, Emerging Concepts and Consensus Recommendations for Aesthetic Use, Including Updates on Complications. Plast Reconstr Surg. 2016;137(3):518e-529e. doi: 10.1097/01.prs.0000475758.63709.23

21. Scherer MA. Specific aspects of a combined approach to male face correction: botulinum toxin A and volumetric fillers. J Cosmet Dermatol. 2016;15(4):566-574. doi: 10.1111/jocd.12247 